\title{
GENDER DIFFERENCES IN THE MOTIVATION OF ENTREPRENEURS IN MODERN COUNTRIES
}

\author{
Iuliia S. Pinkovetskaia \\ Ulyanovsk State University, Ulyanovsk, Russian Federation
}

\begin{abstract}
Entrepreneurship is important in most countries today. One of the main goals of government bodies is to develop and implement measures that ensure an effective gender policy in the development of the business sector, including business motivation. The purpose of the study is to evaluate the values of indicators that characterize the following four options for motivating start-up entrepreneurs, men and women, namely: changing the world for the better, improving well-being and earning higher incomes, continuing the family tradition, the need to earn a living. The study used economic and mathematical modeling based on the development of eight density functions of the normal distribution. The information provided in the Global Entrepreneurship Monitoring Project was considered as initial empirical data. Indicators describing the proportion of different types of motivation typical for men and women who create their own business are studied. The average values of the indicators, as well as their ranges for most countries are determined. A comparative analysis of the values of indicators for Russia and foreign countries is given. The study has a scientific novelty and originality, because it uses economic and mathematical models to evaluate the values of indicators of four variants of entrepreneurial motivation, sets the average values and ranges of changes in the indicators of motivation of start-up entrepreneurs, determines the countries that are characterized by high and low values of the indicators under consideration. The obtained research results have a certain theoretical and applied significance.
\end{abstract} function.

Key words: motivation, women-entrepreneurs, men-entrepreneurs, start-up entrepreneurs, distribution

Citation. Pinkovetskaia I.S. Gender Differences in the Motivation of Entrepreneurs in Modern Countries. Journal of Volgograd State University. Economics, 2020, vol. 22, no. 3, pp. 88-97. (in Russian). DOI: https://doi.org/ 10.15688/ek.jvolsu.2020.3.8

УДК 332.05

Дата поступления статьи: 06.08.2020

ББК 65.09

Дата принятия статьи: 02.09.2020

\section{ГЕНДЕРНЫЕ РАЗЛИЧИЯ В МОТИВАЦИИ ПРЕДПРИНИМАТЕЛЕЙ СОВРЕМЕННЫХ СТРАН}

\author{
Юлия Семеновна Пиньковецкая \\ Ульяновский государственный университет, г. Ульяновск, Российская Федерация
}

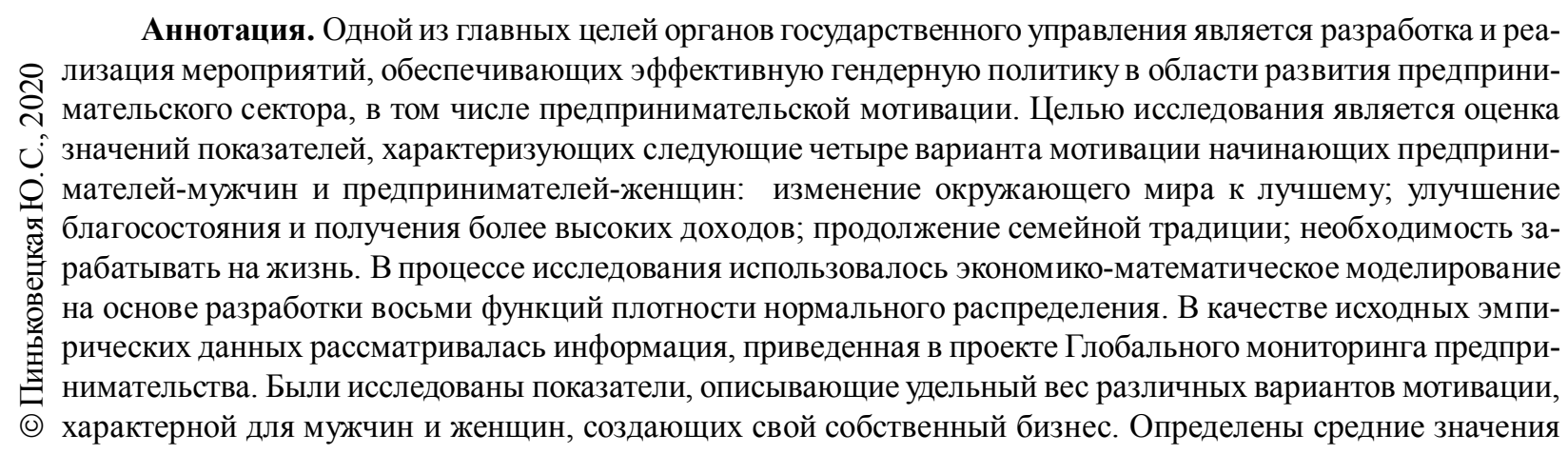


показателей, а также диапазоны их изменения по большинству стран. Приведен сравнительный анализ значений показателей по России и зарубежным странам. В исследовании приведена оценка значений показателей четырех вариантов предпринимательской мотивации; установлены средние значения и диапазоны изменения показателей мотивации начинающих предпринимателей; определены страны с высокими и низкими значениями показателей.

Ключевые слова: мотивация, женщины-предприниматели, мужчины-предприниматели, начинающие предприниматели, функция распределения.

Цитирование. Пиньковецкая Ю. С. Гендерные различия в мотивации предпринимателей современных стран // Вестник Волгоградского государственного университета. Экономика. - 2020. - Т. 22, № 3. - С. 88-97. DOI: https://doi.org/10.15688/ek.jvolsu.2020.3.8

\section{Введение}

Как мужское, так и женское предпринимательство не только играет существенную роль в экономиках современных стран, но и представляется важным для развития личности предпринимателя [The Role of Entrepreneurship ... , 2014]. Известно, что предпринимательский сектор повышает конкурентоспособность и стимулирует экономический рост [De Carolis et al., 2006], обеспечивает занятость для значительной части населения в национальных экономиках [SimonMoya et al., 2016]. Вместе с тем в большинстве стран (особенно развивающихся) оно не получило достаточного развития. Именно поэтому одной из наиболее важных целей, стоящих перед органами государственной власти почти всех стран, является разработка и реализация мероприятий, обеспечивающих эффективную политику в области развития предпринимательского сектора, включающую гендерный аспект. Формирование соответствующих программ требует учета сложившейся мотивации мужчин и женщин, стремящихся стать предпринимателями. Учитывая это, проблема изучения предпринимательской мотивации представляется в настоящее время актуальной.

Развитие предпринимательства требует рассмотрения мотиваций мужчин и женщин, принимающих решения о создании своих собственных бизнесов. Предпринимательская мотивация отражает их стремление к саморазвитию, созданию нового, основанное на внутреннем импульсе [Frese et al., 2014]. При этом предполагается, что все человеческие действия являются результатом как мотивации людей, так и когнитивных факторов, к которым относятся способности, интеллект и навыки [Locke, 2000]. Внешние факторы, а именно состояние национальной экономики, доступность финансовых средств, государственное регулирование, рыночные условия также оказывают существенное влияние на решение стать предпринимателем, по мнению ряда исследователей, именно мотивация людей имеет наибольшее значение на проявление предпринимательской активности [Shane et al., 2012; Huang et al., 2020]. Необходимо отметить, что мотивация влияет на построение стратегии и, соответственно, на успех созданного бизнеса [Gorgievski et al., 2011]. При этом под успехом имеется в виду, соответствует ли этот бизнес ожиданиям предпринимателя.

Мотивация оказывает большое влияние на поведение предпринимателей в процессе их деятельности [Bourlès et al., 2018; Entrepreneurial Success ..., 2016], в том числе укреплении устойчивости своего бизнеса и создании конкурентных преимуществ на рынке.

Предпринимательские мотивации могут изучаться на основе различных научных теорий, в том числе теории предпринимательства [Burton et al., 2016; Van der Zwan et al., 2018; Korsgaard et al., 2011], теории мотивации и стимулирования [Carsrud et al., 2011], теории личности и карьеры [Hall et al., 2005], социальной теории [Robichaud et al., 2010], а также психологии [Piperopoulos et al., 2015].

За последние годы ученые много внимания уделили исследованию мотивации людей, принимающих участие в предпринимательской деятельности. В качестве наиболее важных работ, посвященных вовлечению населения различных стран в предпринимательство, по указанной тематике можно выделить ряд публикаций [Factors Inuencing ... , 2016; Estay et al., 2013; Benz et al., 2008]. Необходимо отметить, что в этих исследованиях основное внимание уделяется таким двум видам мотивации, как добровольная и вынужденная. 
Безусловно, многие люди в качестве причин для начала бизнеса приводят такие мотивы, как желание обеспечить себе и своей семье экономическую безопасность, улучшить благосостояние и получать более высокие доходы [Understanding Motivations ..., 2015].

Ряд научных исследований продемонстрировал связь между предпринимательской деятельностью членов семьи и вероятностью того, что дети и внуки будут создавать собственный бизнес [Solesvik, 2013; Carr et al., 2007]. По мнению исследователей [Schoon et al., 2012], эта тенденция наиболее выражена в мотивации мужчин, которые в процессе проведенного опроса указывали в качестве примера для начала бизнеса своего отца, дедушку или дядю.

Еще одним упоминаемым в публикациях мотивом предпринимательства является необходимость зарабатывать на жизнь, обусловленная сложностями трудоустройства в качестве наемных работников [Necessity Entrepreneurship ... , 2015; Kautonen et al., 2010].

Большинство научных исследований, посвященных мотивации предпринимателей, относятся к экономическому контексту, игнорируя общественные и социальные аспекты, например, такой как желание изменить окружающий мир к лучшему. Вместе с тем этот мотив, наряду с потребностью предпринимателей в повышении своего статуса, самореализации и демонстрации своих достижений, рассмотрен в статьях [The Entrepreneurial Personality ... , 2003; Woldie et al., 2008].

\section{Методика}

Как указывалось выше, исследованиям добровольного и вынужденного предпринимательства в предыдущие годы уделялось значительное внимание. Вместе с тем эта дихотомия не может в полной мере отражать мотивацию начинающих предпринимателей, создающих новые бизнесы. Учитывая это и исходя из анализа публикаций, далее в настоящей статье рассматриваются следующие варианты мотивации мужчин и женщин, считающих целесообразным начать предпринимательскую деятельность:

- изменение окружающего мира к лучшему;

- улучшение благосостояния и получение более высоких доходов;

- продолжение семейной традиции;

- необходимость зарабатывать на жизнь.

В качестве исходной информации в процессе нашего исследования использовались данные, приведенные в проекте Глобального мониторинга предпринимательства (далее ГМП) [Global Entrepreneurship Monitor ... , 2020]. В указанном проекте представлены в том числе значения показателей, характеризующих мотивацию граждан как участвующих в создании нового бизнеса, так и желающих начать эту деятельность в ближайшее время. При этом по каждому из указанных выше вариантов мотивации в проекте ГМП (см. таблицу) приведены значения удельных весов опрашиваемых ранних предпринимателей в об-

Показатели, характеризующие мотивацию РПА, \%

Таблица

\begin{tabular}{|l|c|c|}
\hline \multicolumn{1}{|c|}{ Вариант мотивации } & $\begin{array}{c}\text { Средняя величина } \\
\text { показателя }\end{array}$ & $\begin{array}{c}\text { Диапазон изменения } \\
\text { показателей по большинству } \\
\text { стран }\end{array}$ \\
\hline \multicolumn{1}{|c|}{1} & 2 & 3 \\
\hline Изменение окружающего мира к лучшему, мужчины & 42,39 & $26,03-58,75$ \\
\hline Изменение окружающего мира к лучшему, женщины & 46,03 & $28,92-63,14$ \\
\hline $\begin{array}{l}\text { Улучшение благосостояния и получение более высо- } \\
\text { ких доходов, мужчины }\end{array}$ & 55,51 & $37,83-73,19$ \\
\hline $\begin{array}{l}\text { Улучшение благосостояния и получение более высо- } \\
\text { ких доходов, женщины }\end{array}$ & 49,11 & $30,61-67,61$ \\
\hline Продолжение семейной традиции, мужчины & 36,19 & $18,70-53,68$ \\
\hline Продолжение семейных традиций, женщины & 34,35 & $15,62-53,08$ \\
\hline Необходимость зарабатывать на жизнь, мужчины & 61,57 & $40,08-83,06$ \\
\hline Необходимость зарабатывать на жизнь, женщины & 66,40 & $44,33-88,47$ \\
\hline
\end{tabular}

Примечание. Составлено автором с использованием функций (1)-(8). 
щем их количестве по каждому из вариантов. Отметим, что опрашиваемые предприниматели могли одновременно выбрать несколько вариантов ответов.

В проекте ГМП за 2019 г. приведены соответствующие данные по 50 странам. Географическое расположение этих стран разнообразно. Информация представлена по двадцати одной европейской стране, двум североамериканским странам, восьми латиноамериканским странам, четырнадцати азиатским странам, четырем африканским странам и Австралии. По уровню доходов населения они подразделяются следующим образом: высокие доходы (33 страны), средние доходы (12 стран), низкие доходы (5 стран). Отметим, что в процессе реализации проекта ГМП в каждом из государств проводился опрос не менее двух тысяч начинающих предпринимателей.

В процессе работы нами проверялись следующие гипотезы:

1) значения показателей, характеризующих каждый из вариантов предпринимательской мотивации, существенно отличаются по рассматриваемым странам;

2) значения каждого из показателей не связаны с географическим расположением рассматриваемых стран;

3) значения каждого из показателей не коррелируют с уровнем доходов населения в соответствующих странах.

Для проверки каждой из указанных гипотез разрабатывались экономико-математические модели, представляющие собой функции плотности нормального распределения. Такой методический подход основывается на выполненном ранее автором исследовании [Pinkovetskaia et al., 2018].

Также в процессе работы было осуществлено сравнение значений показателей по России и средних значений по зарубежным странам.

\section{Результаты и обсуждение}

В процессе вычислительного эксперимента нами были разработаны экономикоматематические модели (функции плотности нормального распределения), описывающие закономерности странового распределения четырех показателей, характеризующих мо- тивацию мужчин-предпринимателей и женщин-предпринимателей. Эти функции плотности нормального распределения (y), описывающие распределение значений $(x, \%)$ каждого из четырех мотивов людей, начинающих собственный бизнес по всем рассматриваемым странам приведены ниже:

- изменение окружающего мира к лучшему, мужчины

$$
y_{1}\left(x_{1}\right)=\frac{604,29}{16,36 \cdot \sqrt{2 \pi}} \cdot e^{\frac{-\left(x_{1}-42,39\right)^{2}}{2 \cdot 16,36 \cdot 16,36}}
$$

- изменение окружающего мира к лучшему, женщины

$$
y_{2}\left(x_{2}\right)=\frac{637,86}{17,11 \cdot \sqrt{2 \pi}} \cdot e^{\frac{-\left(x_{2}-46,03\right)^{2}}{2 \cdot 17,11 \cdot 17,11}}
$$

- улучшение благосостояния и получение более высоких доходов, мужчины

$$
y_{3}\left(x_{3}\right)=\frac{528,75}{17,68 \cdot \sqrt{2 \pi}} \cdot e^{\frac{-\left(x_{3}-55,51\right)^{2}}{2 \cdot 17,68 \cdot 17,68}}
$$

- улучшение благосостояния и получение более высоких доходов, женщины

$$
y_{4}\left(x_{4}\right)=\frac{637,86}{18,50 \cdot \sqrt{2 \pi}} \cdot e^{\frac{-\left(x_{4}-49,11\right)^{2}}{2 \cdot 18,50 \cdot 18,50}}
$$

- продолжение семейных традиций, мужчины

$$
y_{5}\left(x_{5}\right)=\frac{678,57}{17,49 \cdot \sqrt{2 \pi}} \cdot e^{\frac{-\left(x_{5}-36,19\right)^{2}}{2 \cdot 17,49 \cdot 17,49}}
$$
женщины

- продолжение семейных традиций,

$$
y_{6}\left(x_{6}\right)=\frac{625,00}{18,73 \cdot \sqrt{2 \pi}} \cdot e^{\frac{-\left(x_{6}-34,35\right)^{2}}{2 \cdot 18,73 \cdot 18,73}} ;
$$
мужчины

- необходимость зарабатывать на жизнь,

$$
y_{7}\left(x_{7}\right)=\frac{833,33}{21,49 \cdot \sqrt{2 \pi}} \cdot e^{\frac{-\left(x_{7}-61,57\right)^{2}}{2 \cdot 21,49 \cdot 21,49}}
$$
женщины 


$$
y_{8}\left(x_{8}\right)=\frac{916,67}{22,07 \cdot \sqrt{2 \pi}} \cdot e^{\frac{-\left(x_{8}-66,40\right)^{2}}{2 \cdot 22,07 \cdot 22,07}} .
$$

Для анализа функций (1)-(8) использовались критерии Колмогорова-Смирнова, Пирсона и Шапиро-Вилка, позволяющие определить качество аппроксимации эмпирических данных. При этом сравнивались расчетные статистики с табличными. Анализ показал высокое качество каждой из восьми функций.

Как указывалось ранее, разработанные функции позволяют установить сложившиеся в странах средние значения показателей ранней предпринимательской активности по рассмотренным одиннадцати отраслям и интервалы их изменения. Они приведены в таблице: средние значения - в столбце 2 , а диапазоны изменения значений показателей по большинству стран - в столбце 3.

Полученные результаты позволяют установить средние значения показателей, а также определить страны с высокими и низкими значениями каждого из восьми показателей, которые оценивались в процессе исследования.

Наиболее высокий уровень мотивов среди ранних предпринимателей в рассматриваемых странах наблюдается в связи с необходимостью зарабатывать на жизнь в соответствующей стране. Такой мотив начала предпринимательской деятельности указали почти две трети всех опрошенных взрослых людей в 50 странах, охваченных проектом ГМП.

По предпринимателям-мужчинам среднее значение показателя достигло почти $62 \%$. В ряде стран на соответствующий мотив ссылались более 83 \% взрослых мужчин. К этим странам относятся Панама, Бразилия, Колумбия (Латинская Америка); Италия (Европа); Армения, Пакистан, Индия, Иордания (Азия); ЮАР, Марокко (Африка). Они характеризуются высокими доходами населения (три страны), средними (четыре страны) и низкими (три страны). Странами, в которых уровень соответствующей мотивации был меньше нижней границы диапазона, приведенного в третьем столбце таблицы, то есть $40 \%$, являются Польша, Нидерланды, Норвегия, Испания, Ирландия, Люксембург (Европа); Япония, Тайвань, Южная Корея (Азия); США (Северная Америка). Они характеризуются высоким уровнем доходов.
По предпринимателям-женщинам среднее значение показателя, характеризующего необходимость зарабатывать на жизнь, достигло $66 \%$. В ряде стран на соответствующий мотив ссылались более $88 \%$ взрослых женщин. К этим странам относятся Панама, Бразилия, Колумбия, Гватемала (Латинская Америка); Армения, Иордания (Азия); Северная Македония, Италия (Европа); ЮАР, Марокко (Африка). По уровню доходов населения страны подразделяются следующим образом: с высокими доходами - три страны, со средними доходами - шесть стран, с низкими доходами - одна страна. Странами, в которых уровень соответствующей мотивации был меньше нижней границы диапазона, приведенного в третьем столбце таблицы, то есть $44 \%$, являются Польша, Норвегия, Нидерланды, Швеция, Люксембург (Европа); Тайвань, Япония, Южная Корея (Азия); Австралия (Австралия). По доходам населения все эти страны характеризуются высоким уровнем.

Около половины взрослых людей в качестве мотивации указали улучшение благосостояния и получение более высоких доходов. Относительно мужчин среднее значение соответствующего показателя составило более $55 \%$. Значения показателя улучшения благосостояния большие, чем верхняя граница диапазона (приведенная в 3-м столбце таблицы), отмечались в таких странах, как Кипр, Италия (Европа); Египет, ЮАР (Африка); Иран, Катар, Пакистан, Индия (Азия). По уровню доходов населения эти страны распределяются следующим образом: высокий (три страны), средний (две страны) и низкий (три страны). Значения показателя меньшие, чем нижняя граница диапазона, были в таких странах, как Польша, Норвегия, Ирландия, Германия, Нидерланды, Словакия (Европа); Мадагаскар (Африка); Эквадор (Латинская Америка). Характеризуются высокими доходами населения (шесть стран), средними (одна страна), низкими (одна страна). Средний уровень этого показателя по предпринимателям-женщинам составил $49 \%$. При этом в таких странах, как Российская Федерация, Белоруссия, Италия (Европа); Израиль, ОАЭ, Катар, Индия, Иран (Азия); ЮАР, Египет (Африка), отмечались значения показателя, превышающие верхнюю границу диапазона. Четыре страны характе- 
ризуются высоким уровнем дохода населения, четыре - средним, одна - низким. Значения показателя меньше нижней границы диапазона (3-й столбец таблицы) отмечаются в таких странах, как Польша, Нидерланды, Норвегия, Швейцария, Словения, Латвия (Европа); Мадагаскар (Африка). Шесть из этих стран расположены в Европе, а одна в Африке. Все расположенные в Европе страны характеризуются высоким уровнем дохода населения, а в Африке - низким.

Более 40 \% начинающих предпринимателей в качестве мотива создания собственного бизнеса выделили изменение окружающего мира к лучшему. При этом в среднем по рассматриваемым странам более $42 \%$ опрошенных мужчин указывали этот мотив. Анализ показал, что к странам с высоким уровнем этого показателя (в соответствии с 3-м столбцом таблицы) относятся США, Канада (Северная Америка); Мексика, Пуэрто-Рико, Панама, Гватемала (Латинская Америка); Люксембург, Польша (Европа); ЮАР (Африка); Индия (Азия). По уровню доходов населения эти страны относятся: шесть стран - высокий, три - средний, одна - низкий. Значения показателя меньшие нижней границы диапазона имели место в Италии, Белоруссии, Российской Федерации (Европа); Южной Корее, Армении, Иордании (Азия); Мадагаскаре, Марокко (Африка). В них отмечается высокий уровень доходов населения в двух странах, средний - в четырех странах, низкий - в двух странах. В среднем по рассматриваемым странам $46 \%$ женщин в качестве мотива начала предпринимательства указали изменение окружающего мира к лучшему. При этом более $63 \%$ женщин определили этот мотив в таких странах, как Мексика, Гватемала, Панама (Латинская Америка); Оман, Индия, Пакистан (Азия); Северная Македония (Европа); США, Канада (Северная Америка); ЮАР (Африка). Они характеризуются высоким уровнем доходов населения - четыре страны, средним - четыре, низким - две. Меньше нижней границы диапазона (3-й столбец таблицы) предпринимательская мотивация женщин в Мадагаскаре, Марокко (Африка); Южной Корее, Армении, Иордании (Азия); Италии, Ирландии, Белоруссии, Российской Федерации (Европа). Они характеризуются высоким уровнем доходов населения - три страны, средним - четыре, низким - две.

Около трети взрослого населения в рассматриваемых странах решили продолжить имеющуюся в их семье традицию предпринимательства. Среди мужчин среднее значение этого показателя в 2019 г. составило 36 \%. Относительно высокий уровень указанного показателя отмечался в Египте (Африка); Германии, Ирландии, Северной Македонии, Польше (Европа); Пакистане, Индии (Азия). В трех странах отмечаются высокие доходы населения, в одной - средние, в трех - низкие. Низкий уровень показателя был в таких странах, как Великобритания, Норвегия, Испания (Европа); Южная Корея, Тайвань (Азия). Все они характеризуются высокими доходами населения. Продолжение семейных традиций является мотивом для $34 \%$ женщин в среднем по рассматриваемым 50 странам. При этом значения больше верхней границы диапазона имели место в Гватемале, Панаме (Латинская Америка); Пакистане, Индии (Азия); Ирландии, Германии, Польше (Европа). В четырех странах отмечаются высокие доходы населения, в одной - средние, в двух низкие. В таких странах, как Южная Корея, Израиль (Азия); Великобритания, Швейцария, Испания, Белоруссия и Нидерланды (Европа), наблюдалась низкая мотивация женщин, решивших продолжить семейную традицию. Эти страны характеризуются высоким уровнем доходов населения - шесть стран, средним - одна.

Необходимо отметить, что средние значения мотивации мужчин по таким показателям, как улучшение благосостояния и получение более высоких доходов, а также продолжение семейной традиции превышали соответствующие значения предпринимательской мотивации женщин. При этом по первому из указанных показателей эта закономерность соблюдается в 30 странах из 50. По показателю продолжение семейной традиции превышение удельного веса мужчин-предпринимателей наблюдается в 31 стране. Противоположная тенденция имела место при сравнении средних значений по показателям изменение окружающего мира к лучшему и необходимость зарабатывать на жизнь. Соответственно, по этим показателям большее количество стран характеризуется преобладани- 
ем женской мотивации над мужской. Так, по показателю изменение окружающего мира к лучшему женщины превалируют в 36 странах из 50 , а по показателю необходимость зарабатывать на жизнь - также в 36 странах.

Представленные в таблице данные, как и их последующее обсуждение, подтвердили правильность трех выдвинутых гипотез.

Представляет интерес изучение данных, характеризующих значения рассматриваемых четырех мотивов раннего предпринимательства по России:

- изменение окружающего мира к лучшему, мужчины - 25,6 \%;

- изменение окружающего мира к лучшему, женщины - 28,7 \%;

- улучшение благосостояния и получение более высоких доходов, мужчины - 70,8 \%;

- улучшение благосостояния и получение более высоких доходов, женщины - 68,5 \%;

- продолжение семейной традиции, мужчины - $26,4 \%$;

- продолжение семейной традиции, женщины $-23,3 \%$;

- необходимость зарабатывать на жизнь, мужчины - 76,0 \%;

- необходимость зарабатывать на жизнь, женщины $-81,7 \%$.

Сравнение этих данных со средними значениями по зарубежным странам позволяет сделать следующие выводы. Первый из рассматриваемых мотивов как у мужчин, так и у женщин в России встречается значительно реже. Это значит, что отечественные предприниматели слабо верят в то, что их деятельность может существенно улучшить жизнь в нашей стране и изменить ее экономику. Второй предпринимательский мотив в опросе российских предпринимателей (мужчин и женщин) упоминался чаще, чем в зарубежных странах. Следовательно, начинающие предприниматели в России больше внимания уделяют проблеме доходности своего бизнеса. Продолжение семейной традиции в нашей стране, по мнению опрошенных мужчин и женщин, реже выступает в качестве мотива начала бизнеса, чем в зарубежных странах. Четвертый предпринимательский мотив в России охватывает более трех четвертей людей, начинающих свой бизнес, в то время как за рубежом этот показатель составляет около двух третьих от количества начинающих предпринимателей (мужчин и женщин).

\section{Выводы и рекомендации}

Задачи, поставленные в ходе исследования, были полностью решены. Выводы исследования, содержащие научную новизну и оригинальность, заключаются в следующем:

- предложено использовать для оценки значений показателей четырех вариантов предпринимательской мотивации экономико-математические модели;

- оценены средние значения и интервалы изменения показателей мотивации начинающих предпринимателей (как мужчин, так и женщин);

- определены страны, для которых характерны высокие и низкие значения рассматриваемых показателей предпринимательской мотивации мужчин и женщин;

- показано, что наибольшие значения показателей, характерны для таких мотивов, как улучшение благосостояния и необходимость зарабатывать на жизнь;

- доказано, что значения каждого из показателей имеют существенную дифференциацию по 50 рассматриваемым странам;

- подтверждено отсутствие зависимости между значениями каждого из восьми показателей и такими факторами, как доходы населения в разных экономиках и их географическое положение.

Полученные результаты исследования имеют определенное теоретическое и прикладное значение. Предложенные показатели и модели их расчета могут быть использованы при обосновании программ развития предпринимательства правительством, региональными и муниципальными органами власти. Итоги расчетов представляют интерес для начинающих предпринимателей. Новые знания о предпринимательской мотивации могут использоваться в деятельности образовательных учреждений.

\section{СПИСОК ЛИТЕРАТУРЫ}

Benz, M. The Value of Doing what You Like: Evidence from the Self-Employed in 23 Countries / M. Benz, B. S. Frey // Journal of Economic Behavior \& Organization. -2008. - Vol. 68 (3). - P. 445-455. 
Bourlès, R. Entrepreneurial Motivation and Business Performance: Evidence from a French Microfinance Institution / R. Bourlès, A. Cozarenco // Small Business Economics. - 2018. - Vol. 51 (4). P. 943-963.

Burton, M. D. A Career Perspective on Entrepreneurship / M. D. Burton, J. B. Sorensen, S. D. Dobrev // Entrepreneurship Theory and Practice. - 2016. - Vol. 40. - P. 237-247.

Carsrud, A. Entrepreneurial Motivations: What Do We Still Need to Know? / A. Carsrud, M. Brannback // Journal of Small Business Management. - 2011. - Vol. 39 (1). - P. 9-26.

Carr, J. C. Prior Family Business Exposure as Intergenerational Influence and Entrepreneurial Intent: A Theory of Planned Behavior Approach / J. C. Carr, J. M. Sequeira // Journal of Business Research. - 2007. - Vol. 60 (10). - P. 1090-1098.

De Carolis, D. M. Social Capital, Cognition, and Entrepreneurial Opportunities: a Theoretical Framework / D. M. De Carolis, P. Saparito // Entrepreneurship Theory and Practice. - 2006. Vol. 1.-P. 41-56.

Entrepreneurial Success Among Single Mothers: The Role of Motivation and Passion / I. Ismail [et al.] // Procedia Economics and Finance. - 2016. Vol. 37 (16). - P. 121-128.

Estay, C. Entrepreneurship: From Motivation to StartUp / C. Estay, F. Durrieu, M. Akhter // Journal of International Entrepreneurship. - 2013. Vol. 11 (3). - P. 243-267.

Factors Inuencing the Entrepreneurial Engagement of Opportunity and Necessity Entrepreneurs / I. Verheul [et al.] // Eurasian Business Review. 2016. - Vol. 6. - P. 273-295.

Frese, M. The Psychology of Entrepreneurship / M. Frese, M. M. Gielnik// Annual Review of Organizational Psychology and Organizational Behavior. - 2014.Vol. 1(1).-P.413-438.

Global Entrepreneurship Monitor 2019-2020. Global Entrepreneurship Research Association (GERA). 2020.

Gorgievski, M. J. 'Small Business Owners' Success Criteria, a Values Approach to Personal Differences / M. J. Gorgievski, M. E. Ascalon, U. Stephan // Journal of Small Business Management. - 2011. - Vol. 49 (2). - P. 207-232.

Hall, D. T. Psychological Success: When the Career is a Calling / D. T. Hall, D. Chandler // Journal of Organizational Behavior. - 2005. - Vol. 26. P. $155-176$.

Huang, Y. Does Entrepreneurial Motivation Help Build Resilience and Facilitate Success in SMEs? - A Study of Founder-Entrepreneurs from High-Tech Software SMEs in China Applied Research / Y. Huang, B. Baruah,
T. Ward / International Conference on Business \& Interdisciplinary Studies (ARICBIS). 1718 February. - L. : [s. n.], 2020.

Kautonen, T. The Impact of a Necessity-Based StartUp on Subsequent Entrepreneurial Satisfaction / T. Kautonen, J. Palmroos // International Entrepreneurship and Management Journal. 2010. - Vol. 6 (3). - P. 285-300.

Korsgaard, S. Enacting Entrepreneurship as Social Value Creation / S. Korsgaard, A. R. Anderson // International Small Business Journal. - 2011. Vol. 2. - P. 135-151.

Locke, E. A. Motivation, Cognition and Action: an Analysis of Studies of Task Goals and Knowledge /E. A. Locke// Applied Psychology: An International Review. -2000. - Vol. 49. -P. 408-429.

Necessity Entrepreneurship and Competitive Strategy / J. Block [et al.] // Small Business Economic. 2015. - Vol. 44. - P. 37-54.

Pinkovetskaia, I. Estimation of Fixed Capital Investment in SMEs: the Existing Differentiation in the Russian Federation / I. Pinkovetskaia, V. Slepova // Business Systems Research. - 2018. Vol. 9 (1). - P. 65-78.

Piperopoulos, P. Burst Bubbles or Build Steam? Entrepreneurship Education, Entrepreneurial Self-Efficacy, and Entrepreneurial Intentions / P. Piperopoulos, D. Dimov // Journal of Small Business Management. - 2015. - Vol. 53 (4). P. 970-985.

Robichaud, Y. Necessity and Opportunity-Driven Entrepreneurs in Canada: An Investigation into Their Characteristics and an Appraisal of the Role of Gender / Y. Robichaud, R. LeBrasseur, K. V. Nagarajan // The Journal of Applied Business and Economics. - 2010. - Vol. 11 (1). P. 59-79.

Schoon, I. Who Becomes an Entrepreneur? Early Life Experiences as Predictors of Entrepreneurship / I. Schoon, K. Duckworth // Developmental Psychology. - 2012. - Vol. 48 (6). - P. 1719-1726.

Shane, S. Entrepreneurial Motivation / S. Shane, E. A. Locke, C. J. Collins // Human Resource Management Review. - 2012. - Vol. 13 (2). P. 257-279.

Simon-Moya, V. Influence of Economic Crisis on New SME Survival: Reality or Fiction? / V. SimonMoya, L. Revuelto-Taboada, D. Ribeiro-Soriano // Entrepreneurship and Regional Development. 2016. - Vol. 28, iss. 1-2. - P. 157-176.

Solesvik, M. Z. Entrepreneurial Motivations and Intentions: Investigating the Role of Education Major / M. Z. Solesvik // Education + Training. 2013. - Vol. 55 (3). - P. 253-271.

The Entrepreneurial Personality in the Context of Resources, Environment, and the Startup 
Process - A Configurational Approach /C. Korunka [et al.] // Entrepreneurship Theory and Prasctice. - 2003. - Vol. 28 (1). - P. 23-42.

The Role of Entrepreneurship in US Job Creation and Economic Dynamism / R. Decker [et al.] // Journal of Economic Perspectives. - 2014. - Vol. 3. P. 3-24.

Understanding Motivations for Entrepreneurship / U. Stephan [et al.] // BIS Research paper No. 212. Department for Business, Innovation and Skills Drews IFF Research. L. : [s. n.], 2015. -109 p.

Van der Zwan, P. W. Self-Employment and Satisfaction with Life, Work, and Leisure / P. W. Van der Zwan, J. Hessels, C. A. Rietveld // Journal of Economic Psychology. - 2018. - Vol. 64. - P. 73-88.

Woldie, A. Factors Influencing Small and Medium Enterprises (SMEs): An Exploratory Tudy of Owner Manager and Firm Characteristics / A. Woldie, P. Leighton, A. Adesua // Banks and Bank Systems. - 2008. - Vol. 3 (3). - P. 5-13.

\section{REFERENCES}

Benz M., Frey B.S. The Value of Doing What You Like: Evidence from the Self-Employed in 23 Countries. Journal of Economic Behavior \& Organization, 2008, vol. 68 (3), pp. 445-455.

Bourlès R., Cozarenco A. Entrepreneurial Motivation and Business Performance: Evidence from a French Microfinance Institution. Small Business Economics, 2018, vol. 51 (4), pp. 943-963.

Burton M.D., Sorensen J.B., Dobrev S.D. A Career Perspective on Entrepreneurship. Entrepreneurship Theory and Practice, 2016, vol. 40, pp. 237-247.

Carsrud A., Brannback M. Entrepreneurial Motivations: What Do We Still Need to Know? Journal of Small Business Management, 2011, vol. 39 (1), pp. 9-26.

Carr J.C., Sequeira J.M. Prior Family Business Exposure as Intergenerational Influence and Entrepreneurial Intent: A Theory of Planned Behavior Approach. Journal of Business Research, 2007, vol. 60 (10), pp. 1090-1098.

De Carolis D.M., Saparito P. Social Capital, Cognition, and Entrepreneurial Opportunities: A Theoretical Framework. Entrepreneurship Theory and Practice, 2006, vol. 1, pp. 41-56.

Ismail I., Husin N., Rahim N.A., Kamal M.H.M., Mat R.C. Entrepreneurial Success Among Single Mothers: The Role of Motivation and Passion. Procedia Economics and Finance, 2016, vol. 37 (16), pp. 121-128.

Estay C., Durrieu F., Akhter M. Entrepreneurship: From Motivation to Start-Up. Journal of International Entrepreneurship, 2013, vol. 11(3), pp. 243-267.
Verheul I., Thurik R., Hessels J., Zwan P. Factors Influencing the Entrepreneurial Engagement of Opportunity and Necessity Entrepreneurs. Eurasian Business Review, 2016, vol. 6, pp. 273-295.

Frese M., Gielnik M.M. The Psychology of Entrepreneurship. Annual Review of Organizational Psychology and Organizational Behavior, 2014, vol. 1 (1), pp. 413-438.

Global Entrepreneurship Monitor 2019-2020. Global Entrepreneurship Research Association (GERA). 2020.

Gorgievski M.J., Ascalon M.E., Stephan U. Small Business Owners Success Criteria, a Values Approach to Personal Differences. Journal of Small Business Management, 2011, vol. 49 (2), pp. 207-232.

Hall D.T., Chandler D. Psychological Success: When the Career Is a Calling. Journal of Organizational Behavior, 2005, vol. 26, pp. 155-176.

Huang Y., Baruah B., Ward T. Does Entrepreneurial Motivation Help Build Resilience and Facilitate Success in SMEs? - A Study of FounderEntrepreneurs from High-Tech Software SMEs in China Applied Research. International Conference on Business \& Interdisciplinary Studies (ARICBIS). 17-18 February. London, [s. n.], 2020.

Kautonen T., Palmroos J. The Impact of a NecessityBased Start-Up on Subsequent Entrepreneurial Satisfaction. International Entrepreneurship and Management Journal, 2010, vol. 6 (3), pp. 285-300.

Korsgaard S., Anderson A.R. Enacting Entrepreneurship as Social Value Creation. International Small Business Journal, 2011, vol. 2, pp. 135-151.

Locke E.A. Motivation, Cognition and Action: An Analysis of Studies of Task Goals and Knowledge. Applied Psychology: An International Review, 2000, vol. 49, pp. 408-429.

Block J., Kohn K., Miller D., Ullrich K. Necessity Entrepreneurship and Competitive Strategy. Small Business Economic, 2015, vol. 44, pp. 37-54.

Pinkovetskaia I., Slepova V. Estimation of Fixed Capital Investment in SMEs: The Existing Differentiation in the Russian Federation. Business Systems Research, 2018, vol. 9(1), pp. 65-78.

Piperopoulos P., Dimov D. Burst Bubbles or Build Steam? Entrepreneurship Education, Entrepreneurial Self-Efficacy, and Entrepreneurial Intentions. Journal of Small Business Management, 2015, vol. 53 (4), pp. 970-985.

Robichaud Y., LeBrasseur R., Nagarajan K.V. Necessity and Opportunity-Driven Entrepreneurs in Canada: An Investigation into Their Characteristics and an Appraisal of the Role of 
Gender. The Journal of Applied Business and Economics, 2010, vol. 11 (1), pp. 59-79.

Schoon I., Duckworth K. Who Becomes an Entrepreneur? Early Life Experiences as Predictors of Entrepreneurship. Developmental Psychology, 2012, vol. 48 (6), pp. 1719-1726.

Shane S., Locke E.A., Collins C.J. Entrepreneurial Motivation. Human Resource Management Review, 2012, vol. 13 (2), pp. 257-279.

Simon-Moya V., Revuelto-Taboada L., Ribeiro-Soriano D. Influence of Economic Crisis on New SME Survival: Reality or Fiction? Entrepreneurship and Regional Development, 2016, vol. 28, iss. 1-2, pp. 157-176.

Solesvik M.Z. Entrepreneurial Motivations and Intentions: Investigating the Role of Education Major. Education + Training, 2013, vol. 55 (3), pp. 253-271.

Korunka C., Frank H., Lueger M., Mugle J. The Entrepreneurial Personality in the Context of Resources, Environment, and the Startup
Process - A Configurational Approach. Entrepreneurship Theory and Practice, 2003, vol. 28 (1), pp. 23-42.

Decker R., Haltiwanger J., Jarmin R., Miranda J. The Role of Entrepreneurship in US Job Creation and Economic Dynamism. Journal of Economic Perspectives, 2014, vol. 3, pp. 3-24.

Stephan U., Hart M., Mickiewicz T., Drews C.-C. Understanding Motivations for Entrepreneurship. BIS Research Paper No. 212. Department for Business, Innovation and Skills Drews IFF Research. London, [s.n.], 2015. 109 p.

Van der Zwan P.W., Hessels J., Rietveld C.A. SelfEmployment and Satisfaction with Life, Work, and Leisure. Journal of Economic Psychology, 2018, vol. 64, pp. 73-88.

Woldie A., Leighton P., Adesua A. Factors Influencing Small and Medium Enterprises (SMEs): An Exploratory Tudy of Owner Manager and Firm Characteristics. Banks and Bank Systems, 2008, vol. 3 (3), pp. 5-13.

\section{Information About the Author}

Iuliia S. Pinkovetskaia, Candidate of Sciences (Economics), Associate Professor, Department of Economic Analysis and State Management, Ulyanovsk State University, L. Tolstogo St, 42, 432000 Ulyanovsk, Russian Federation, judy54@yandex.ru, https://orcid.org/0000-0002-8224-9031

\section{Информация об авторе}

Юлия Семеновна Пиньковецкая, кандидат экономических наук, доцент кафедры экономического анализа и государственного управления, Ульяновский государственный университет, ул. Л. Толстого, 42, 432000 г. Ульяновск, Российская Федерация, judy54@yandex.ru, https://orcid.org/0000-0002-8224-9031 